\title{
Ambiguity in 3-D patterns induced by lighting assumptions
}

\author{
Thomas V Papathomas \\ Laboratory of Vision Research and Department of Biomedical Engineering, Rutgers University, \\ New Brunswick, NJ 08903, USA
}

\section{Andrei Gorea}

Laboratoire de Psychologie Experimentale, CNRS, Université René Descartes, 28 rue Serpente, 75006 Paris, France

Received 23 January 1990, in revised form 20 September 1990

\begin{abstract}
A bistable pattern is shown with white and black bars with horizontal and vertical orientations which produce an impression of thin slabs stacked up in depth either toward or away from the observer. It is postulated that the ambiguity is induced by the observer's assumption of the direction of the light source.
\end{abstract}

In our efforts to study the roles that orientation and luminance polarity play in texture segregation (Gorea and Papathomas 1989, 1990; Papathomas and Gorea 1990; Papathomas et al 1990), we almost accidentally produced the images shown in figure 1 , in which white and black bars with horizontal and vertical orientations form staircase patterns. These patterns produce vivid impressions of three-dimensional objects arranged in depth. One possible percept is that of thin slabs, stacked on top of each other. Another is that of 3-D steps or stepped rows of seats.

The arrangement of both the top and bottom halves of figure 1a seems to have two possible stable interpretations: as we traverse the path shown by the arrow, the slabs are stacked up either toward or away from us. These two stable states seem to alternate either spontaneously or at will for trained observers, as we shall discuss shortly. As a result, when one views figure 1a in its entirety, the top and bottom halves may induce either a coherent global percept or, as most of our casual observers noticed, a conflicting one; in the latter case, the observer fails to perceive figure $1 \mathrm{a}$ as a single, coherent, 3-D structure.

By contrast, the two halves of figures $1 \mathrm{~b}$ and $1 \mathrm{c}$, which are identical with figure $1 \mathrm{a}$ but merely rotated by $90^{\circ}$ counterclockwise and clockwise, respectively, always produce a dominant stable interpretation, as the reader may verify. Figure 1b (or 1c) forms the impression of a convex (or concave) solid angle along the vertical border, in which the slabs are stacked toward the observer in the direction of the arrow.

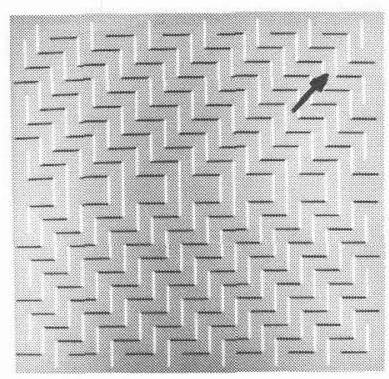

(a)

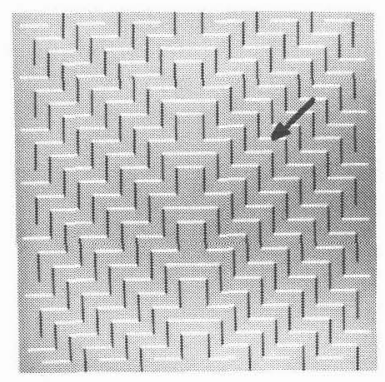

(b)

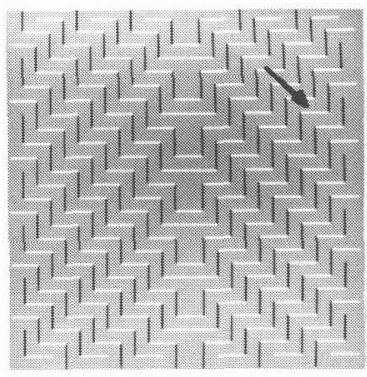

(c)

Figure 1. Staircase patterns referred to in the text. 
One way that figure $1 \mathrm{~b}$ can be visualized, for example, is as an edge of an ancient pyramid when viewed from above. In addition to the dominant percept in figures $1 \mathrm{~b}$ and $1 \mathrm{c}$, there is a latent percept in each, not easily obtained, in which the angles become concave and convex, respectively.

One possible explanation, which accounts for all the percepts described above, is in terms of the assumption the observer makes on the position of the light source. The images of figure 1 can be thought of as parallel (not perspective) projections of the structure shown in figure $2 \mathrm{a}$, which attempts to depict part of the top half of figure 1a. The three principal unit vectors are shown for reference, with $\hat{z}$ coming out of the page (right-handed coordinate system). If we assume that the parallel light rays (coming from a source located an infinite distance away) have a strong component in the $-\hat{\boldsymbol{x}}$ direction, a weak component in the $-\hat{z}$ direction, and no component in the $\hat{y}$ direction, then the orthographic projections along lines of sight $-\hat{z}$ (front view) and $-\hat{\boldsymbol{x}}$ (side view) would be as shown in figures $2 \mathrm{~b}$ and $2 \mathrm{c}$, respectively.

In this interpretation, the image-plane normal, ie the direction in which the eye is located, is along the vector $(\hat{\boldsymbol{x}}, \hat{\boldsymbol{y}}, \hat{\boldsymbol{z}})$. The percept is that slab $\mathrm{A}$ is in front and occludes part of the slab B which, in turn, partially occludes slab C. The volume of each slab lies below the staircase contour. Parenthetically, we have assumed Lambertian surfaces, which diffuse the incident light in all directions, with the intensity of the reflected light proportional to $\cos u$, where $u$ is the angle between the surface normal and the vector pointing to the light source (this explains the gray, black, and white intensities).

However, if we assume the light source in the opposite direction and we go through an analysis similar to that outlined by figure 2 (but with a different coordinate system), we now get the second stable percept in which slab $\mathrm{C}$ is in front and partially occludes slab B, which, in turn, occludes part of slab A; here, however, the volume of each slab lies above the staircase contour.

Our explanation of the bistable percept is that, in the case of figure $1 \mathrm{a}$, both directions of the light source (from the left or from the right) are equally likely and equally easy to be adopted by the observer, either spontaneously or at will. Experienced observers can switch between the two percepts at will and all of them report that the easier way to do that is by deliberately assuming the light source in one of two opposing directions. However, most of the naive observers report their inability to perceive figure $1 \mathrm{a}$ as a coherent structure. In order to experience such a

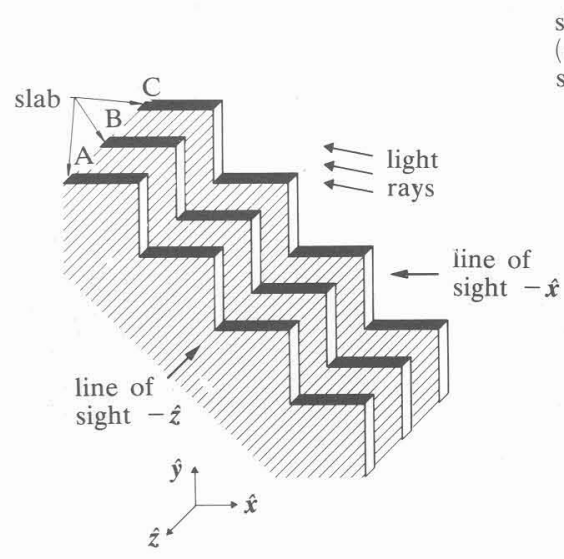

(a)

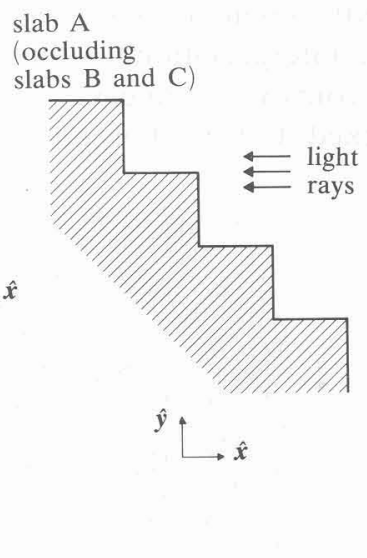

(b)

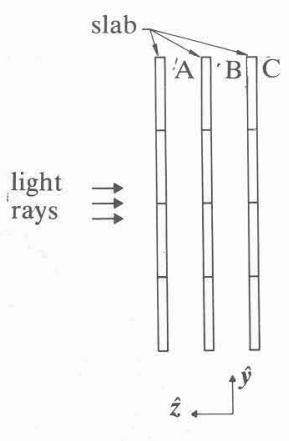

(c)

Figure 2. (a) The structure shown in top half of figure 1a, and (b) and (c) its parallel projections. 
coherent whole, the observer (or his/her visual system) needs to assume the existence of two, mutually exclusive, light sources. We conjecture that this is not a natural task for the visual system.

By contrast, the inherently dominant assumption of a source shining from above, a consequence of living on Earth with the Sun overhead (Brewster 1847; Kaufman 1974; Yonas et al 1979; Ramachandran 1988a), produces the stable percept described earlier for figures $1 \mathrm{~b}$ and $1 \mathrm{c}$. In fact, the suppressed percept may be obtained in figures $1 \mathrm{~b}$ and $1 \mathrm{c}$, perhaps with difficulty, if one assumes a light source shining from below. In both cases, however, the assumption of a single light source is necessary (Ramachandran 1988b). We mention parenthetically that it is much more difficult to obtain the suppressed percept in images composed of shaded hemispherical shapes, which can be perceived either as convex or concave (see, for example, figure 2 in Ramachandran 1988a). This may be attributed to the strong impression of 3-D smooth surfaces created by such highly realistically shaded images, in which it is very difficult for the viewer to override the assumption that the light source is located overhead.

Additional evidence for the validity of our explanation is that when the gray background and the black and white lines of figure 1 are replaced by a black background and equiluminant red and green lines, respectively, the impression of a threedimensional structure is almost entirely eliminated. Perhaps there are other factors which may help to explain the visual experience elicited by our stimuli, but we believe that our explanation of the percepts as a result of lighting assumptions adds an interesting example to that class of phenomena.

The interesting stimuli described in this paper have been used in the design of the logo of the 13th European Conference on Visual Perception (held in Paris in September 1990), reproduced in the announcement of the Conference which appeared in this journal (1989, Perception 18 695).

\section{References}

Brewster D, 1847 "On the conversion of relief by inverted vision" Edinburgh Philosophical Transactions 15657

Gorea A, Papathomas T V, 1989 "The role of color and orientation matching in texture discrimination" Optical Society of America, 1989 Annual Meeting Technical Digest 18161

Gorea A, Papathomas T V, 1990 "Texture segregation by chromatic and achromatic visual pathways: an analogy with motion processing" Journal of the Optical Society of America 8 $386-393$

Kaufman L, 1974 Sight and Mind: An Introduction to Visual Perception (New York: Oxford University Press) p. 228

Papathomas T V, Gorea A, 1990 "The role of visual attributes in texture perception" paper presented at the 1990 SPIE-SPSE Symposium on Human Vision and Visual Processing, Santa Clara, California

Papathomas T V, Gorea A, Julesz B, 1990 "Juxtaposition of orientation, luminance and polarity in perceptual grouping" Investigative Opthalmology and Visual Science 31105

Ramachandran V S, 1988a "Perception of shape from shading" Nature (London) 331 163-166

Ramachandran V S, 1988b "Perceiving shape from shading" Scientific American $25676-83$

Yonas A, Kuskowski M, Sternfels S, 1979 "The role of frames of reference in the development of responsiveness to shading" Child Development 50 495-500 